\title{
Tratamento da dor orofacial através da acupuntura em pacientes com bruxismo: um estudo de revisão
}

\section{Orofacial pain treatment through acupuncture in patients with bruxism: a review study}

\author{
Joelson Santos* \\ Pamella Recco ${ }^{* *}$ \\ Guilherme Mota** \\ Antônio Vinícius Holanda ${ }^{* * * *}$ \\ Valdeci Elias dos Santos Junior ${ }^{* * * *}$
}

\section{Resumo}

O bruxismo é um hábito parafuncional que consiste em ranger e apertar os dentes, envolvendo movimentos rítmicos, semelhantes ao da mastigação, causando fadiga muscular e dor. As dores orofaciais sempre foram causa de preocupação na odontologia, portanto é constante a busca por novos tratamentos terapêuticos, como por exemplo, a acupuntura. Objetivo: o objetivo desse trabalho foi realizar uma revisão de literatura com ênfase nos mecanismos pelos quais a acupuntura pode se mostrar eficaz na analgesia de pacientes com bruxismo, descrevendo as principais bases científicas dessa técnica. Materiais e método: a estratégia de busca das publicações incluiu as bases de dados Medline, Lilacs e Pubmed. A seleção dos artigos foi feita com base nos seguintes critérios: ter sido escrito em inglês, espanhol ou português; apresentar resumo estruturado e abordar aspectos que relacionem o mecanismo de ação e a efetividade da acupuntura sobre a dor oriunda de bruxismo. Considerações finais: o mecanismo de ação da acupuntura ainda não está claro, mas verifica-se que com a aplicação desta técnica há uma liberação endógena de diversos mediadores químicos, como os opióides, acetilcolina e cortisona, influenciando na percepção da dor. Desta forma, essa terapia representa uma alternativa terapêutica no tratamento da dor facial crônica e miofacial, como as provenientes do bruxismo.

Palavras-chave: Acupuntura. Dor facial. Bruxismo.

\section{Introdução}

Atualmente observa-se na prática clínica um considerável aumento da incidência do bruxismo associado a outras alterações do sistema estomatognático ${ }^{1}$. O bruxismo, que consiste no hábito parafuncional de ranger os dentes, constitui um grande desafio para o cirurgião-dentista, pois se trata de uma desordem funcional que exige uma participação multidisciplinar no sentido de reconhecer os fatores etiológicos e promover benefícios ao indivíduo portador dessa patologia ${ }^{2}$.

É importante que os profissionais que trabalham com pacientes que apresentam e sofrem com as consequências das desordens do sistema estomatognático e estruturas relacionadas tenham consciência de que também os fatores somáticos, psíquicos e sociais podem estar alterados e comprometer o senso normal de bem estar ${ }^{2}$.

O bruxismo é o resultado de um distúrbio neuromotor dos músculos mastigatórios, especificamente o bruxismo do sono caracteriza-se por ranger os dentes durante o sono e, geralmente, está associado com despertares de curta duração, conhecidos como microdespertares ${ }^{3}$. O bruxismo noturno difere do bruxismo diurno por envolver: distintos estados de consciência, além de diferentes estados fisiológicos. Essa desordem funcional também pode ser clas-

\footnotetext{
Aluno de Graduação em Odontologia - Faculdade Integrada de Pernambuco, Recife-PE, Brasil.

Professora Adjunta da Faculdade Integrada de Pernambuco - Facipe, Recife-PE, Brasil.

Especialista em acupuntura e Mestrando Universidade Federal de Pernambuco, Recife-PE, Brasil.

Doutor e Professor Titular da Faculdade Integrada de Pernambuco, Recife-PE, Brasil.

Professor Adjunto da Faculdade de Odontologia da Universidade Federal de Alagoas, Maceió-AL, Brasil.
} 
sificada como primária ou secundária, o bruxismo secundário está associado com transtornos clínicos e/ou psiquiátricos, diferente do primário ${ }^{4-6}$.

Dessa forma, o hábito do bruxismo pode alterar alguns componentes do sistema estomatognático. A nível muscular, na maioria dos casos, pode gerar dor e sensibilidade à palpação. Em relação à articulação temporomandibular pode ocorrer dores e ruídos, devido à perda da coordenação dos dois feixes do músculo pterigóideo lateral ${ }^{7,8}$.

O tratamento do bruxismo é bastante variável e depende da etiologia, dos sinais observados ao exame físico e dos sintomas relatados pelo paciente. Dentre os tratamentos para dores oriundas dessa disfunção, destaca-se a acupuntura, que devido às suas propriedades antinflamatórias, ansiolíticas, miorrelaxantes e ativadoras da função imunológica, tem se apresentado como terapia alternativa, integrativa, complementar e efetiva ${ }^{9}$.

Sendo assim, o objetivo desse trabalho foi, por meio de uma revisão de literatura, entender o mecanismo de ação da acupuntura e sua eficácia para o tratamento de dores orofaciais proveniente de bruxismos.

\section{Metodologia}

A investigação dos artigos incluídos neste estudo foi realizada nas bases de dados Medline, Lilacs e Pubmed, com a utilização dos seguintes descritores de saúde em inglês: Bruxism, Acupuncture e Facial Pain. Foram considerados todos os estudos publicados sobre o tema até junho de 2017.

Os critérios de inclusão dos artigos foram: ter sido escrito em inglês, espanhol ou português, apresentar resumo estruturado e resultados que abordassem o mecanismo de ação e a efetividade da acupuntura sobre a dor oriunda de bruxismo.

Os critérios de exclusão estabelecidos foram: artigos que não se enquadraram nos critérios de inclusão expostos ou ensaios clínicos com ausência de um grupo de controle.

A busca se procedeu utilizando a combinação dos descritores e do descritor booleano "AND". Os filtros "Clinical trial" e "Humans" foram utilizados para delimitar a pesquisa. "As estratégias para a busca foram: "Bruxism AND Acupuncture" e "Acupunture AND Facial pain". Um total de 44 artigos foram encontrados.

Para a busca utilizando os descritores "Bruxism AND Acupuncture" em adição aos filtros foram encontrados 2 estudos. Porém, um estudo foi excluído por estar no idioma italiano.

Utilizando-se dos descritores "Acupunture AND Facial pain" com os filtros adicionados anteriormente foram encontrados 42 estudos. Seis estudos foram rejeitados por estarem em idioma diferentes dos citados nos critérios de inclusão. Onze artigos foram rejeitados devido ao uso de terapêuticas com- binadas com a acupuntura. Outros onze artigos foram rejeitados por não ter relação com bruxismo. Por fim, mais dois estudos não foram incluídos por não apresentar resumos estruturados.

\section{Discussão}

Este trabalho teve por objetivo verificar a eficácia da acupuntura nas dores provenientes de bruxismo, bem como detalhar o mecanismo de ação dessa técnica milenar chinesa.

A acupuntura faz parte da medicina tradicional Chinesa, sendo um método que visa prevenir e/ou tratar a dores por meio da inserção de agulhas em diferentes locais anatômicos do corpo, correspondendo a pontos específicos denominados acupontos ${ }^{1}$ Sob a perspectiva biológica, bioquímica e neurofisiológica, existem vários estudos que explicam o efeito da acupuntura. Esses estudos mostram que no momento da inserção da agulha de acupuntura, uma série de mediadores químicos reguladores da dor são libertadas para a corrente sanguínea ( $\beta$-endorfina, endorfina, seretonina, dopamina $)^{10-14}$.

A persistência e recorrência da dor pode afetar a qualidade de vida das pessoas com bruxismo, verificando-se alterações a nível da ansiedade, limitações funcionais e aumento da irritabilidade. Assim, a acupuntura surge como uma ferramenta alternativa para o tratamento de dores derivadas dessa disfunção, reestabelecendo a qualidade de vida das pessoas $^{12,14}$.

\section{Mecanismo de ação}

Villalobos et al. ${ }^{15}$ (2006) relataram que a acupuntura atua no eixo hipotálamo-pituitário-adrenal, através da ativação de centros corticais e da liberação de substâncias antinflamatórias e analgésicas. A inserção das agulhas nos pontos de acupuntura atua sobre os receptores nociceptivos gerando um potencial de ação elétrico e um pequeno processo inflamatório local. Ocorrem assim a liberação dos neurotransmissores bradicina e histamina, e os estímulos são conduzidos ao SNC pelas fibras A-delta, espessas e mielinizadas, e fibras C, finas e amielínicas, localizadas na pele e nos músculos.

As fibras A $\delta$ (A-delta) estimulam os neurônios encefalinérgicos por meio de sinapses e liberam encefalina, bloqueador da substância P (neurotransmissor que estimula a dor), inibindo assim a sensação dolorosa. Os estímulos continuam até o tronco encefálico, liberando serotonina, que será responsável pelo aumento dos níveis de endorfina e de ACTH (hormônio adrenocorticotrófico) e, consequentemente, diminuição de cortisol, garantindo assim o efeito benéfico da acupuntura no estresse e na ansiedade do paciente ${ }^{16}$.

A inserção das agulhas de acupuntura desempenham três efeitos locais: elétrico, neuroquímico, por 
ação mecânica, e misto que corresponde a uma associação dos outros dois. Além da serotonina e endorfina liberadas, são produzidos também histamina, íons potássio e prostaglandinas, que estimulam os quimiorreceptores, diminuindo o limiar excitatório. O potencial de ação da membrana, desencadeado pela inserção de uma agulha de acupuntura metálica deve-se à ação da liberação de substâncias causadas pela lesão traumática celular local ${ }^{17}$.

Nesse contexto, quando os pontos de acupuntura são estimulados, ocorre ativação das fibras nervosas mielínicas encontradas nos músculos, que enviam o impulso para a medula espinhal ativando assim o mesencéfalo, o hipotálamo e a hipófise, causando a liberação de encefalina, beta-endorfina, dinorfina, serotonina e noradrenalina no organismo resultando na inibição da sensação dolorosa ${ }^{18}$.

\section{Acupuntura e dor orofacial oriunda do bruxismo}

O bruxismo do sono é considerado um distúrbio de movimento relacionado ao sono. Essa parafunção é caracterizada pelo contato não-funcional dos dentes, que pode ocorrer de forma consciente ou inconsciente, manifestando-se pelo ranger ou apertar dos mesmos. Não é uma doença, mas quando exacerbada pode levar a um desequilíbrio fisiopatológico do sistema estomatognático. Várias modalidades terapêuticas têm sido sugeridas, mas não há um consenso sobre qual é a mais eficiente ${ }^{4-6}$.

A acupuntura vem se tornando cada vez mais popular e aceita, devido aos seus efeitos positivos sobre o alívio da dor aguda e crônica. O uso da acupuntura em pacientes com dor oriunda do bruxismo é eficaz para o aumento da amplitude de movimentos mandibulares, proporcionado pelo relaxamento dos músculos mastigatórios e uma analgesia de início lento, contudo duradoura ${ }^{19-21}$.

Dessa forma, sugere-se que a atuação da acupuntura sobre o controle da dor ocorre por ativação das vias opióides e não-opióides. Esse estímulo proporciona a ativação do sistema modulador da dor, através do aumento da estimulação das terminações nervosas de fibras mielínicas A, que são responsáveis por conduzir o estímulo para os centros medulares, encefálicos e eixo hipotálamo-hipófise. Já na medula espinhal, o estímulo acontece por inibição pré-sináptica, devido à liberação de encefalinas e dinorfinas ${ }^{22}$.

A técnica de acupuntura consiste em estimular determinados pontos do corpo, que possuem uma resistência elétrica diferente dos tecidos que os rodeiam. De acordo com a medicina tradicional chinesa, o corpo humano é percorrido por canais de energia que conectam os diversos órgãos entre si e sobre esses canais localizam-se os pontos de acupuntura que somam aproximadamente 750. A literatura sugere para o tratamento do bruxismo os pontos E6
(Jiache) e TA17 (Yifeng) e os pontos TA17, B20, VB21 para tratar a tensão muscular facial ${ }^{7}$.

A acupuntura pode ser utilizada como terapia alternativa para o controle da dor no tratamento de pacientes com bruxismo, pois se trata de um método terapêutico que é capaz de diminuir a atividade dos músculos mastigatórios em repouso nos pacientes portadores de cefaleia miogênica ${ }^{23}$. A elevação dos níveis de excreção de catecolaminas na urina está relacionada a estados de estresse e ansiedade, que por sua vez são agentes desencadeantes do bruxismo. A redução dos níveis de neurotransmissores catecolinérgicos, após sessões de acupuntura confirmam a relação existente entre a técnica e tal mediador químico, o que justifica a indicação dessa terapia para esse tipo de parafunção ${ }^{24,25}$.

Com o objetivo de avaliar a eficácia da acupuntura sobre o acuponto IG4 (Hegu) no tratamento do bruxismo noturno, em relação aos sintomas associados à dor dos músculos da mandíbula, foram realizadas avaliações subjetivas da dor na cabeça e no pescoço antes e após o tratamento, e então, verificada uma redução significativa da dor na região mandibular, assim como diminuição da tensão na face ${ }^{26}$. Um estudo conduzido por Blasco e Martín ${ }^{26}$ (2017) relatou uma melhora no quadro álgico e nos sintomas derivados do bruxismo do sono após a estimulação de pontos de gatilhos para a dor no masseter e temporal. A redução dos sintomas do bruxismo e da dor foram, respectivamente, de forma imediata e após 7 dias de terapia.

Usualmente as placas miorrelaxantes vêm sendo usadas como forma de tratamento para o bruxismo do sono. Dessa forma, a acupuntura foi comparada à terapia com placas miorelaxantes para dores orofaciais oriundas de bruxismo. Ambas as terapêuticas foram eficazes no alívio de quadros álgicos, porém a acupuntura foi mais efetiva para dores na região temporal $^{27-29}$. Segundo Shen e Goddard ${ }^{30}$ (2007), isso acontece devido ao aumento da tolerância à dor dos músculos mastigatórios após acupuntura.

Rancam et al. ${ }^{31}$ (2009) demonstraram que a acupuntura foi capaz de causar uma melhora em aproximadamente $60-75 \%$ dos pacientes com dor crônica e portador de bruxismo do sono e que pode também ser utilizada com o objetivo de prevenção e tratamento em diversas desordens crânio-faciais. Além disso, a acupuntura raramente provoca reações adversas, o que torna a técnica segura para pacientes com hipertensão, diabetes, cardiopatias, alergias, além de poder ser usada em crianças, gestantes e pacientes com necessidades especiais ${ }^{32-34}$.

\section{Considerações finais}

Segundo a literatura consultada, a acupuntura tem se mostrado uma alternativa para o controle da dor orofacial oriunda do bruxismo. O mecanismo de ação da acupuntura ainda não está totalmente elu- 
cidado, mas as evidências científicas atuais apontam que com o uso da técnica ocorre a liberação de mediadores químicos que modulam a dor.

\section{Abstract}

Bruxism is a parafunctional habit consisting of grinding and clenching teeth, involving rhythmic movements similar to chewing, and causing muscle fatigue and pain. Orofacial pain has always been a cause of concern in Dentistry, so there is a constant search for new therapeutic treatments such as acupuncture. Objective: this study aimed to conduct a literature review focusing on mechanisms through which acupuncture may prove itself effective for orofacial analgesia of patients with bruxism, describing the main scientific bases of this technique. Materials and method: the search strategy for publications included Medline, Lilacs, and Pubmed databases. Studies were selected based on the following criteria: have been written in English, Spanish, or Portuguese, present structured abstract, and address issues that relate the action mechanism and acupuncture effectiveness on the pain caused by bruxism. Final considerations: the action mechanism of acupuncture is still unclear, but it appears that the application of this technique causes an endogenous release of several chemical mediators such as opioids, acetylcholine, and cortisone, influencing the perception of pain. Thus, acupuncture is a therapeutic alternative for the treatment of chronic facial pain and myofascial pain, such as those from bruxism.

Keywords: Acupuncture. Facial pain. Bruxism.

\section{Referências}

1. Gama E, Andrade AO, Campos RM. Bruxismo: Uma revisão da literatura. Ciência Atual-Revista Científica Multidisciplinar das Faculdades São José, 2013; 1:115-23.

2. Cordon R, Sesma N, Laganá, DC. Odontologia Multidisciplinar: O Paciente no Centro das Atenções. Elsevier Brasil; 2015 .

3. American sleep disorders association. International classification of sleep disorder: diagnostic and coding manual, revised. 2nd ed. Westchester, 2005; 89-192.

4. Bader G, Lavigne G. Sleep bruxism; an overview of na oromandibular sleep movement disorder. Sleep Med Rev, London 2000;4(1):27-43.

5. Chase M H, Morales F R. Control of motoeurons during sleep. In: Principles and practice of sleep medicine. Philadephia: WB Saunders; 2000. p.155-68.

6. Kato T, Montplaisir J Y, Guitard F, Sessle B J, Lund J P, Lavigne G J. Evidence that experimentally induced sleep bruxism is a consequence of transient arousal. J Dent Res. 2003;82(4):284-8.

7. Calderan MF; Thiago Cruvinel Silva; Daniela Rios Honório; Thais Marchini Oliveira; Maria Aparecida de Andrade Moreira Machado et al. Fatores etiológicos do bruxismo do sono: revisão de literatura. Rev. Odontol. Univ Cid São Paulo (Online) 2015; 26(3):115-21.

8. Alfaya TA; TANNURE PN; Roberta BARCELOS; Etyene Castro DIP; Luciana UEMOTO; Cresus Vinícius Depes
GOUVÊA et al. Clinical management ofchildhoodbruxism. RGO 2015; 63(2):207-12.

9. Dallanora LJ; Pedro Paulo Feltrin, Ricardo Tatsuo Inove; Vânia Aranha dos Santos; Julio Tanaka et al. Avaliação do uso de acupuntura no tratamento de pacientes com bruxismo. RGO 2004; 52(5):333-9.

10. Cho S, Whang W. Acupuncture for Temporomandibular Disorders: A Systematic Review. J Orofac Pain 2010; 24(2):152-62.

11. Rosted P. Practical recommendations for the use of acupuncture in the treatment of temporomandibular disorders based on the outcome of published controlled studies. Oral Diseases 2001; 7:109-15.

12. Wang M, Loo WTY, Chou JWK. Electromyographic responses from the stimulation of the temporalis muscle through facial acupuncture points. J Chiropract Med 2007; 6:146-52.

13. Taguchi A, Sharma N, Ali SZ, Dave B, Sessler DI, Kurz A. The effect of auricular acupuncture on anaesthesia with desflurane. Anaesthesia 2002; 57: 1159-63.

14. Myers CD, White BA, Heft MW. A review of complementary and alternative medicine use for treating chronic facial pain. JADA 2002; 133: 1189-96.

15. Villalobos YC, Hidalgo SH, Gomez SMD, Navarro IC. Eficacia de la acupuntura enel síndrome dolordisfuncióndel aparato temporomandibular. Archivomédico de Camaguey 2006;10(5):4.

16. Rosted P. Introduction to acupuncture in dentistry.Br Dent J 2000; 189:136-40

17. Sant'anna CBM; Zuim PRJ; Guidotti AM; Brandini DA; Dos Santos DM; Turcio KHL et al. Acupuntura como tratamento de dor muscular: um estudo preliminar. Revista de Odontologia da UNESP. 2014;43:120-5.

18. Da Silva G, Eliane Castilhos Corrêa; Ana Maria Toniolo da Silva; Jovana Moura Milanesi1 et al. Acupuntura como recurso terapêutico na dor e na gravidade da desordem temporomandibular. Fisioterapia e Pesquisa 2011; 18(3):217-22.

19. Charlton E. Ethical guidelines for pain research in humans. committee on Ethical issues of the international association for the Study of Pain. Pain 1995; 63:277-8.

20. Grillo CM, Meirelles MPMR, Sousa MLR. Tratamento da disfunção temporomandibular com acupuntura: relato de caso clínico. Rev Paul Odontol 2010; 32(1):31-3.

21. Smith P, Mosscrop D, Davies S, Sloan P, Al-Ani Z. The efficacy of acupuncture in the treatment of temporomandibular joint myofascial pain: a randomised controlled trial. J Dent. 2007 35(3):259-67.

22. Jensen LB; Tallgren A; Troest T; Jensen SB et al. Effect of acupuncture on myogenic headache. Scand J Dent Res 1977; 85(6):456-70

23. Jensen LB. Effect of acupuncture on tension headache and urinary catecholamine excretion.Scand J Dent Res 1982; 90(5):397-403

24. List T, Helkimo M, Andersson S, Carlsson GE. Acupuncture and occlusal splint therapy in the treatment of craniomandibular disorders. Part I. A comparative study. Swed Dent J. 1992;16(4):125-41

25. Koo ST; KS Lim, K Chung, H Ju, JM Chung et al. Electroacupuncture-induced analgesia in a rat model of ankle sprain pain is mediated by spinal alpha-adrenoceptors. Pain 2008; 135(1-2):11-9.

26. Blasco-Bonora PM, Martín-Pintado-Zugasti A. Effects of myofascial trigger point dry needling in patients with sleep bruxism and temporomandibular disorders: a prospective case series. Acupunct Med 2017; 35(1):69-74.

27. Turo D, Otto P, Hossain M, Gebreab T, Armstrong K, Rosenberger WF et al. Novel Use of Ultrasound Elastography to Quantify Muscle Tissue Changes After Dry Needling of 
Myofascial Trigger Points in Patients With Chronic Myofascial Pain. J Ultrasound Med 2015 ; 34(12):2149-61.

28. List T, Helkimo M, Andersson S, Carlsson GE. Acupuncture and occlusal splint therapy in the treatment of craniomandibular disorders. Part I. A comparative study. Swed Dent J 1992; 16(4):125-41.

29. Johansson A, Wenneberg B, Wagersten C, Haraldson T. Acupuncture in treatment of facial muscular pain. Acta Odontol Scand 1991; 49(3):153-8.

30. Shen YF, Goddard G. The short-term effects of acupuncture on myofascial pain patients after clenching. Pain Pract 2007; 7(3):256-64.

31. Rancam; César Bataglion; Solange Aparecida Bataglion; Odinê Maria Rêgo Bechara; Marisa Semprini; Selma Siéssere; João Paulo Machado de Sousa; José Alexandre de Souza Crippa; Jaime Eduardo Cecilio Hallak; Simone Cecilio Hallak Regalo et al. Acupunture and temporomandibular disorders: a 3-moth follow-up EMG study. The Journal of Alternative and complementary Medicine 2009; 15(12) 1307-10.

32. Vianna RS; Alice Granthon de Souza; Bruna Cunha da Silva; Teresa Ávila Berlinck; Kátia Regina Hostílio Cervantes Dias et al. A acupuntura e sua aplicação na odontologia. UFES Ver Odontol. 2008; 10 (4):48-52.

33. Santos LMM, Martelete M.; J MANICA - MANICA Acupuntura no tratamento da dor. In: MANICA, J. et al. Anestesiologia. Princípios e técnicas. 3. ed. Porto Alegre: Artmed; 2004. p.1307-1309.

34. Simma I, Gleditsch JM, Simma L, Piehslinger E. Immediate effects of microsystem acupuncture in patients with oromyofacial pain and craniomandibular disorders (CMD): a double-blind, placebo-controlled trial. Br Dent J 2009; 207(12):E26.

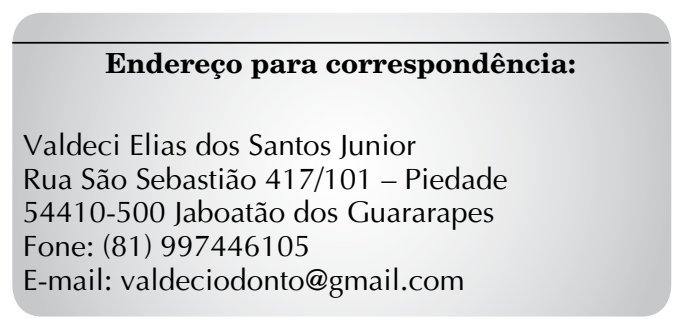

Recebido: 09/11/2016. Aceito: 02/03/2017. 